\title{
An artificial neural network model for rainfall forecasting in Bangkok, Thailand
}

\author{
N. Q. Hung, M. S. Babel, S. Weesakul, and N. K. Tripathi \\ School of Engineering and Technology, Asian Institute of Technology, Thailand \\ Received: 14 December 2007 - Published in Hydrol. Earth Syst. Sci. Discuss.: 30 January 2008 \\ Revised: 29 June 2009 - Accepted: 27 July 2009 - Published: 7 August 2009
}

\begin{abstract}
This paper presents a new approach using an Artificial Neural Network technique to improve rainfall forecast performance. A real world case study was set up in Bangkok; 4 years of hourly data from 75 rain gauge stations in the area were used to develop the ANN model. The developed ANN model is being applied for real time rainfall forecasting and flood management in Bangkok, Thailand. Aimed at providing forecasts in a near real time schedule, different network types were tested with different kinds of input information. Preliminary tests showed that a generalized feedforward ANN model using hyperbolic tangent transfer function achieved the best generalization of rainfall. Especially, the use of a combination of meteorological parameters (relative humidity, air pressure, wet bulb temperature and cloudiness), the rainfall at the point of forecasting and rainfall at the surrounding stations, as an input data, advanced ANN model to apply with continuous data containing rainy and non-rainy period, allowed model to issue forecast at any moment. Additionally, forecasts by ANN model were compared to the convenient approach namely simple persistent method. Results show that ANN forecasts have superiority over the ones obtained by the persistent model. Rainfall forecasts for Bangkok from 1 to $3 \mathrm{~h}$ ahead were highly satisfactory. Sensitivity analysis indicated that the most important input parameter besides rainfall itself is the wet bulb temperature in forecasting rainfall.
\end{abstract}

\section{Introduction}

Accurate information on rainfall is essential for the planning and management of water resources. Additionally, in the urban areas, rainfall has a strong influence on traffic, sewer

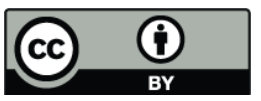

Correspondence to: N. Q. Hung

(nguyenquang.hung@ ait.ac.th) systems, and other human activities. Nevertheless, rainfall is one of the most complex and difficult elements of the hydrology cycle to understand and to model due to the complexity of the atmospheric processes that generate rainfall and the tremendous range of variation over a wide range of scales both in space and time (French et al., 1992). Thus, accurate rainfall forecasting is one of the greatest challenges in operational hydrology, despite many advances in weather forecasting in recent decades (Gwangseob and Ana, 2001).

Neural networks have been widely applied to model many of nonlinear hydrologic processes such as rainfall-runoff (Hsu et al., 1995; Shamseldin, 1997), stream flow (Zealand et al., 1999; Campolo and Soldati, 1999; Abrahart and See, 2000), groundwater management (Rogers and Dowla, 1994), water quality simulation (Maier and Dandy, 1996; Maier and Dandy, 1999), and rainfall forecasting. More detailed discussion regarding the concepts and applications of ANN in hydrology can be referred to in the two technical papers prepared by the ASCE Task Committee on Application of Artificial Neural Networks in Hydrology as appeared in the Journal of Hydrologic Engineering (ASCE, 2000). A pioneer work in applying ANN for rainfall forecasting was undertaken by French et al. (1992), which employed a neural network to forecast two-dimensional rainfall, $1 \mathrm{~h}$ in advance. Their ANN model used present rainfall data, generated by a mathematical rainfall simulation model, as an input data. This work is, however, limited in a number of aspects. For example, there is a trade-off between the interaction and the training time, which could not be easily balanced. The number of hidden layers and hidden nodes seem insufficient, in comparison with the number of input and output nodes, to reserve the higher order relationship needed for adequately abstracting the process. Still, it has been considered as the first contribution to ANN's application and established a new trend in understanding and evaluating the roles of ANN in investigating complex geophysical processes. 
Toth et al. (2000) compared short-time rainfall prediction models for real-time flood forecasting. Different structures of auto-regressive moving average (ARMA) models, artificial neural networks and nearest-neighbors approaches were applied for forecasting storm rainfall occurring in the Sieve River basin, Italy, in the period 1992-1996 with lead times varying from 1 to $6 \mathrm{~h}$. The ANN adaptive calibration application proved to be stable for lead times longer than 3 hours, but inadequate for reproducing low rainfall events.

Koizumi (1999) employed an ANN model using radar, satellite and weather-station data together with numerical products generated by the Japan Meteorological Agency (JMA) Asian Spectral Model and the model was trained using 1-year data. It was found that the ANN skills were better than the persistence forecast (after $3 \mathrm{~h}$ ), the linear regression forecasts, and the numerical model precipitation prediction. As the ANN model was trained with only 1 year data, the results were limited. The author believed that the performance of the neural network would be improved when more training data became available. It is still unclear to what extent each predictor contributed to the forecast and to what extent recent observations might improve the forecast.

Past studies have obviously indicated that ANN is a good approach and has a high potential to forecast rainfall. The ANN is capable to model without prescribing hydrological processes, catching the complex nonlinear relation of input and output, and solving without the use of differential equations (Luk et al., 2000; Hsu et al., 1995; French et al., 1992). In addition, ANN could learn and generalize from examples to produce a meaningful solution even when the input data contain errors or is incomplete (Luk et al., 2000). Most of the existing ANN models applied in rainfall forecasting are event based; the models were fed in input with screened/generated data that contained only rainy periods (i.e., rainfall events, rainy days or monthly rainfall data). By using only the data from the rainy periods as training data, ANN models could easily identify the patterns characterizing the rainfall. However, on the other hand, any features or characteristics not included within the training data will not be learned by ANN. Translated this means that conventional ANN models could only issue accurate forecasting when rain occurred already and they can estimate how long the rain would last, but they are unable to predict whether it would rain or not if there is no rain at the time of issuing forecast. Hence, most of the previous studies of ANN on rainfall forecast are not fully suitable for the application in real time forecasting.

The main objective of this study is to develop a rainfall forecast model using ANN technique. The developed ANN model is designed to run a real time task, in this situation, the input to the model should not be event based data but consecutive data including non-rainy periods to acquire a fully representation of both rain and no rain conditions. When using only continuous past rainfall data to train an ANN model, no rain periods with zero values make no change in weights update process so the ANN could not recognize the pattern and provided poor forecasting results. Targeting to explore alternative ways to overcome this problem, several models were tested by changing model architecture, transfer function and employing additional data as input variables. Results of preliminary test showed that with the use of additional data (meteorology data and rainfall record from surrounding stations), a continuous ANN model could perform highly accuracy of rainfall forecast and can be used for real time applications. Applying the developed ANN model, rainfall from 1 to $6 \mathrm{~h}$ ahead was forecasted at 75 rain gauge stations (as forecast point) in Bangkok city, Thailand, using present hourly rainfall data and meteorological parameters of relative humidity, air pressure, wet bulb temperature, cloudiness, and rainfall from surrounding rain gauge stations as input variables. Additionally, along with the ANN model, a persistent model was developed and compared with the predictions of the ANN model in order to reveal the real advantage of the continuous ANN model in term of real time forecast. Sensitivity analysis is also carried out to identify the most and least important factors in predicting rainfall in the study area.

\section{Study area}

Bangkok, the capital and commercial city of Thailand, is one of the highly developed cities in Southeast Asia. Having a land area of $1569 \mathrm{~km}^{2}$, it is located in the central part of the Thailand on the floodplain of the Chao Phraya River, with latitude $13.45 \mathrm{~N}$ and longitude $100.35 \mathrm{E}$. The area has a tropical type of climate with long hours of sunshine, high temperatures and high humidity. There are three main seasons; rainy (April-October), winter (November-January) and summer (February-March). The average minimum temperature is approximately in low to mid $20 \mathrm{~s}^{\circ} \mathrm{C}$ and high temperature in mid $30 \mathrm{~s}^{\circ} \mathrm{C}$. Bangkok receives an average annual rainfall of $1500 \mathrm{~mm}$ and is influenced by the seasonal monsoon. The city is affected by the flood on regular basis due to rainfall, which paralyzes most of the daily activities. Some of the immediate consequences of a heavy rainfall in Bangkok are: water clogging in the streets, heavy traffic jams, blackouts, and direct or indirect economic losses.

The flood events in Bangkok occur from two sources: the rainfall and the rise in water level in the Chao Phraya River due to large flow from upstream. In the past, most of the occurrence of high river flow and heavy rains in the city resulted in severe flooding. However, with the construction of dams in the upstream and a dike along the riverbank in Bangkok, nearly all parts of the city are now protected from flooding. Land use in Bangkok has changed rapidly in the last few decades and development or urbanization of the area has increased the impervious land, increasing flood volume and frequency. The construction of drainage infrastructure has not kept pace with the land-use change due to lack of investment in the sector. This has resulted in reduced capacity of the drainage system. In addition, lack of hydrological 


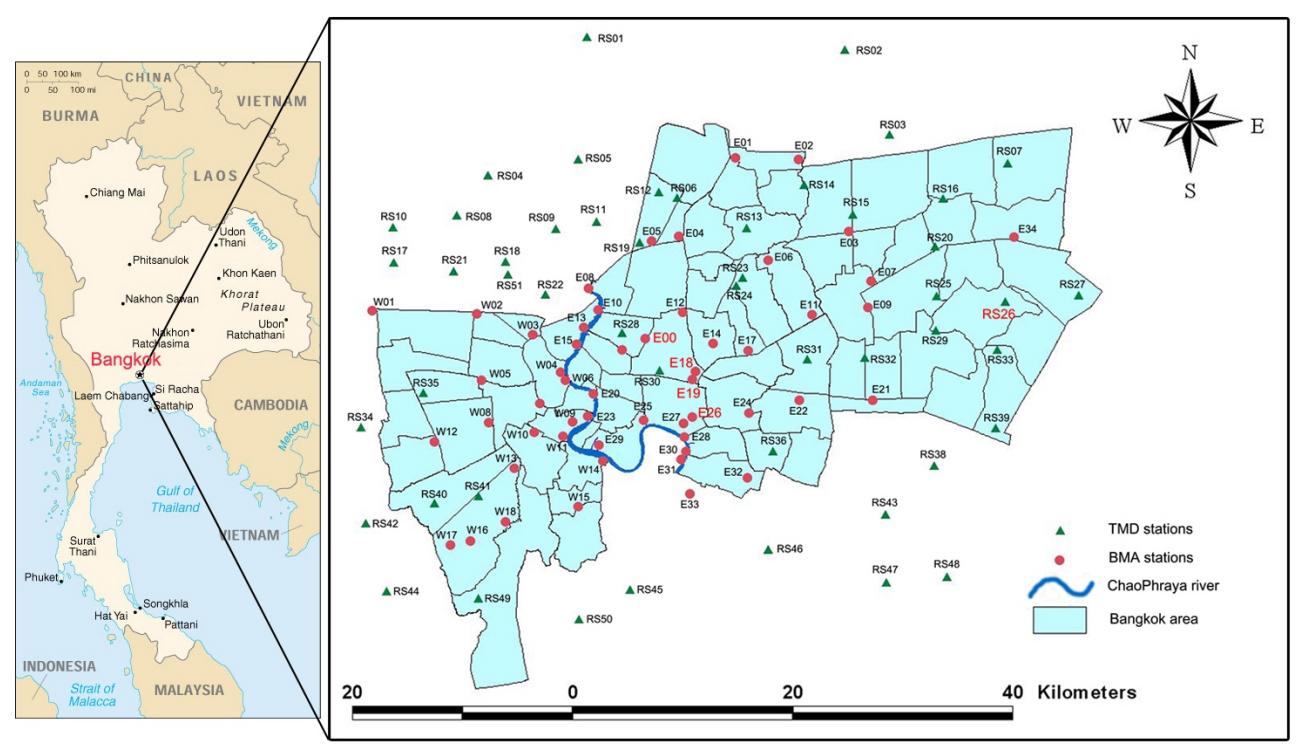

Fig. 1. Location of the study area and the rain gauge stations network.

information and the failure of gravity to effectively remove drainage water from the city make urban flooding inevitable during the wet season. For the city like Bangkok, one of the best ways to cope with the flooding problem is to provide advance rainfall forecasting and flood warning. Knowing the condition of rainfall in Bangkok in advance can help in managing and dealing with problems due to flooding.

The Department of Drainage and Sewage (DDS) of the Bangkok Metropolitan Administration (BMA) had established the Bangkok Metropolitan Flood Control Center (FCC) in 1990 for systematic and efficient management of operation and control of flood protection facilities. The BMA has 53 online rain gauge stations scattered throughout Bangkok and sensors installed at canal gates and pumping stations that observe water level. The observed data is transferred in real time to the FCC by ultra high frequency radio signals every $15 \mathrm{~min}$. Besides, the Thai Meteorological Department (TMD) owns a network of 51 rain gauge stations covering Bangkok and nearby areas. Both rain gauge networks consist of rain gauges of tipping bucket type with $0.5 \mathrm{~mm}$ accuracy. Furthermore, Bangkok has one 100-m tall meteorological mast station covered the area. Data collected at the meteorological station was transmitted via a dedicated telephone line to a central processing computer for data storage and analysis. These data are now available on the Internet and can be used for online applications. Locations of the rain gauges are shown in Fig. 1.

There was actually no reliable rainfall forecast mechanism using rain gauge data in the past. Based upon the historical data and the current situation, the flood forecast analysis is manually carried out at the FFC. After a decision about control policy is made out of this analysis, the flood control protection command is then broadcasted to all remote control stations (gates and pumping stations). This system is acceptable in terms of real time data transmission but not efficient in terms of urban flood forecast and flood management. Therefore, there is a need to investigate and apply an accurate technique for real time rainfall forecasting. ANN with its advantages such as computation speed, learning capability, fault tolerance and adoptability, has been selected to be a tool for short-term rainfall forecast using rain gauge data for Bangkok area. The model is mimic design, so it can be applied not only to Bangkok area but also to other tropical developing urban areas as well.

Historical rainfall data was collected from 104 stations of the BMA and TMD rain gauge networks for the period from 1991 to 2005 . After analyzed data, the period from 1 January 1997 to 31 December 1999 was selected to train ANN models, and the data of the year 2003 were used as a testing set. This study focus on the Bangkok area only, so total 75 stations inside Bangkok area were selected, while the other 29 stations which are located outside Bangkok were discarded, it roughly made each selected station representing for an area around $21 \mathrm{~km}^{2}$. The collected meteorological data which contained hourly measurements of six parameters observed in the mast station, that is: relative humidity, wet bulb temperature, dry bulb temperature, air pressure, cloudiness, and wind speed for the same period as rainfall data. As an additional variable, the average hourly rainfall intensity of all the rain gauges was simply arithmetically average computed and provided with the meteorology data.

Figure 2 shows the average monthly rainfall, taken over a period from 1991 to 2003, in Bangkok. There are two peaks of rainfall during a year, the first in May, and the second in October. The meteorological data of 1991-2004 shows that the average annual relative humidity (RH) is about $81 \%$ with 


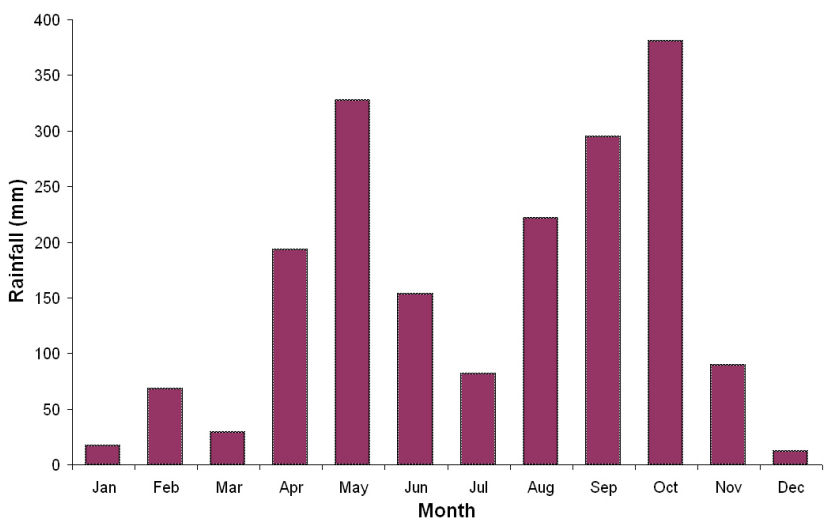

Fig. 2. Average monthly rainfall in Bangkok.

the average maximum $\mathrm{RH}$ of $93 \%$ and the average minimum $\mathrm{RH}$ of $52 \%$. The average annual temperature is $26.8^{\circ} \mathrm{C}$, with the average maximum temperature of $33.4^{\circ} \mathrm{C}$ in April and the average minimum temperature of $20.4^{\circ} \mathrm{C}$ in December. The average annual rainfall is $1869.5 \mathrm{~mm}$ with the highest average monthly rainfall of approximately $381 \mathrm{~mm}$ observed in October, and the lowest average monthly rainfall of about $12 \mathrm{~mm}$ occurring in December, usually the driest month of the year.

\section{Artificial neural network}

An artificial neural network (ANN) is an interconnected group of artificial neurons that has a natural property for storing experiential knowledge and making it available for use. The first simplest form of feedforward neural network, called perceptron has been introduced by Rosenblatt in 1957. This original perceptron model contained only one layer, inputs are fed directly to the output unit via the weighted connections. Although the perceptron initially seemed promising, it was eventually proved that perceptrons could not be trained to recognize many classes of patterns. After that, multilayer perceptron (MLP) model was derived in 1960 and gradually became one of the most widely implemented neural network topologies. Multilayer perceptron means a feedforward network with one or more layers of nodes between the input and output nodes. The MLP overcomes many limitations of the single layer perceptron, their capabilities stem from the non-linear relationships among the nodes (Lippmann, 1987). In theirs study of nonlinear dynamics, Lapedes and Farber (1987) have pointed out the important that the MLP is capable of approximating arbitrary functions. Two important characteristics of the MLP are: its nonlinear processing elements (PEs) which have a nonlinearity that must be smooth (the logistic function and the hyperbolic tangent are the most widely used); and their massive interconnectivity (i.e. any element of a given layer feeds all the elements of the next layer).
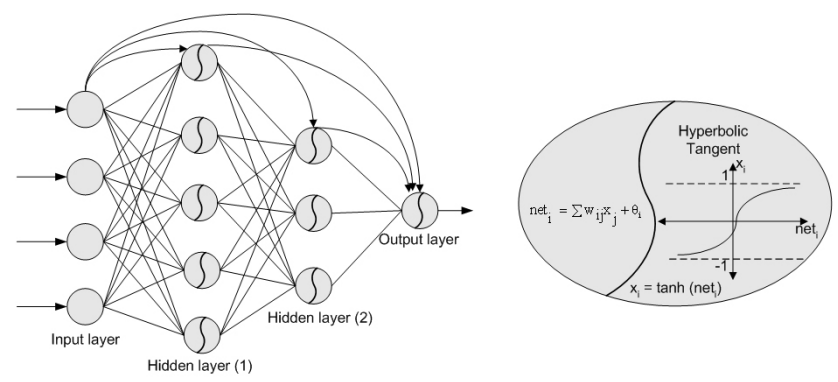

Fig. 3. A simple generalized feedforward neural network with hyperbolic tangent function.

Generalized feedforward networks are a generalization of the MLP such that connections can jump over one or more layers. In theory, a MLP can solve any problem that a generalized feedforward network can solve. In practice, however, generalized feedforward networks often solve the problem much more efficiently. A classic example of this is the two-spiral problem. Without describing the problem, it suffices to say that a standard MLP requires hundreds of times more training epochs than the generalized feedforward network containing the same number of processing elements. A simple generalized feedforward neural network with two hidden layers is shown in Fig. 3.

An optimal ANN architecture may be considered as the one yielding the best performance in terms of error minimization, while retaining a simple and compact structure. There are two important issues concerning the implementation of artificial neural networks, that is, specifying the network architecture (the number of nodes and layers in the network) and finding the optimal values for the connection weights (selection of a training algorithm). In the process of specifying the network size, an insufficient number of hidden nodes causes difficulties in learning data whereas an excessive number of hidden nodes might lead to unnecessary training time with marginal improvement in training outcome as well as make the estimation for a suitable set of interconnection weights more difficult (Zealand et al., 1999). A higher number of nodes in hidden layer tend the network to memorize, instead of learning and generalization, and it might lead to the problem of local minima. On the other hand, increasing the hidden nodes will help to adjust to larger fluctuation of target function and allow the model to consider the presence of volatilities in the data. Such as trends and seasonal variation often appear a lot with rainfall. There is actually no specific rule to determine the appropriate number of hidden nodes; yet the common method used is trial and error based on a total error criterion. This method starts with a small number of nodes, gradually increasing the network size until the desired accuracy is achieved. Fletcher and Goss (1993) proposed a suggestion number of node in the hidden layer ranging from $(2 n+1)$ to $(2 \sqrt{n}+m)$ where $n$ is the number of 
input node, and $m$ is the number of output node. The number of input and output nodes is problem-dependent, and the number of input nodes depends on data availability. In addition, the selection of input should be based on priori knowledge of the problem. A firm understanding of the hydrologic system under consideration is necessary for the effective selection of input data (Ahmad and Simonovic, 2005).

Regarding the second issue, several training processes are available to find the values of connection weights. These algorithms differ in how the weights are obtained. The selection of training algorithm is related to the network type, computer memory, and the input data. There are several training algorithms which can be randomly listed as follow: QuickProp (QP), Orthogonal Least Square (OLS), LevembergMarquart (LM), Back Propagation (BP), and Resilient Propagation Algorithm (RPROP). As implied in this study, the standard back propagation algorithm is used in ANN training based on its most popular success, Coulibaly et al. (2000) stated that $90 \%$ of ANN models applied in the field of hydrology used the back propagation algorithm. In fact the renewed interest in ANN was in part triggered by the existence of back propagation which was first introduced by Werbos in 1974 for the three layer perceptron network. The application area of the MLP network remained rather limited until the breakthrough in 1986 when Rumelhart and McClelland published theirs work with back propagation and gained recognition. The back propagation rule propagates the errors through the network and allows adaptation of the hidden units. This algorithm involves minimizing the global error by using the steepest descent or gradient approach. The network weights and biases are adjusted by incrementing the negative gradient of the error function for each iteration.

The multilayer perceptron is trained with error-correction learning, which means that the desired response for the system must be known. The error correction learning works in the following way: from the system response $d_{i}(n)$ at node $i$ at iteration $n$, and the desired response $y_{i}(n)$ for a given input pattern, an instantaneous error $e_{i}(n)$ is defined by

$e_{i}(n)=d_{i}(n)-y_{i}(n)$

Using the theory of gradient-descent learning, each weight in the network can be adapted by correcting the present value of the weight with a term that is proportional to the present input and error at the weight, i.e. the weight from node $j$ to node $i\left(w_{i j}\right)$ can be calculated by:

$w_{i j}(n+1)=w_{i j}(n)+\eta \delta_{i}(n) x_{j}(n)$

where, $x_{j}$ is a transform function at node $j, i$ and $j$ indicate different layers.

The local error $\delta_{i}(n)$ can be directly computed from $e_{i}(n)$ at the output node or can be computed as a weighted sum of errors at the internal nodes. The constant $\eta$ is called the step size. Most gradient search procedures require the selection of step size. The larger step size, the faster the minimum can be reached. However, if the step size is too large, then the algorithm will diverge and the error will increase instead of decrease. If the step size is too small then it will take too long to reach the minimum, which also increases the probability of getting caught in local minima. Momentum learning is an improvement to the straight gradient descent in the sense that a memory term (the past increment to the weight) is used to speed up and stabilize convergence. In momentum learning the equation to update the weights becomes

$w_{i j}(n+1)=w_{i j}(n)+\eta \delta_{i}(n) x_{j}(n)+\alpha\left(w_{i j}(n)-w_{i j}(n-1)\right)(3)$

where $\alpha$ is the momentum. Normally $\alpha$ should be set between 0.1 and 0.9 . The back propagation algorithm is applied as follow:

1. Initialize all weights and bias (normally a small random value) and normalize the training data.

2. Compute the output of neurons in the hidden layer and in the output layer (net) using

$n e t_{i}=\sum w_{i j} x_{i}+\theta_{i} \quad ; \quad \theta_{i}$ is a bias for $P E_{i}$

1. Compute the error and weight update.

2. Update all weights, bias and repeat steps 2 and 3 for all training data.

3. Repeat steps 2 to 4 until the error converges to an acceptable level.

\section{ANN models in preliminary test}

In the model designing stage, several models were first tested at one station in order to find the optimal ANN model that can then be employed for all other stations in the forecasting stage. The station E18, located in the Sukhumvit area, where a real-time flood forecasting system is currently being developed, was chosen as a sample station to design the ANN model set up. Split-sample training is a common method to train ANN models; in this method, collected data are divided into a training and testing set. However, recent works have found that the better-trained model is not always coupled with better performance in the testing. A practical way to find a point of better generalization and avoid over training is to set aside a small percentage of the training data set, which then can be used for cross validation. Monitoring the errors in the training set and the validation set should be carried out during the training process. When the error in the validation set increases, the training should stop because the point of best generalization has reached. This cross validation approach was adopted for the training of ANN models in this study. Accordingly, three years data $(1997,1998,1999)$ with hourly rainfall records were selected to train ANN models, in which $80 \%$ of the data was set as a training set and $20 \%$ of the data was set aside to use for cross validation. The 
Table 1. Alternative ANN models considered in the study.

\begin{tabular}{lllll}
\hline Model & Network type & Transfer function & Architecture & Training data \\
\hline $\mathrm{A}$ & Simple multilayer perceptron & Sigmoid & $5-5-5-1$ & $\mathrm{R}_{t-4}, \mathrm{R}_{t-3}, \mathrm{R}_{t-2}, \mathrm{R}_{t-1}$, and $\mathrm{R}_{t}$ \\
$\mathrm{~B}$ & Simple multilayer perceptron & Sigmoid & $5-10-10-1$ & $\mathrm{R}_{t-4}, \mathrm{R}_{t-3}, \mathrm{R}_{t-2}, \mathrm{R}_{t-1}$, and $\mathrm{R}_{t}$ \\
$\mathrm{C}$ & Generalized feedforward & Sigmoid & $5-10-10-1$ & $\mathrm{R}_{t-4}, \mathrm{R}_{t-3}, \mathrm{R}_{t-2}, \mathrm{R}_{t-1}$, and $\mathrm{R}_{t}$ \\
$\mathrm{D}$ & Generalized feedforward & Sigmoid & $6-16-12-1$ & $\mathrm{R}_{t}, \mathrm{RH}_{t}, \mathrm{WBT}_{t}, \mathrm{AP}_{t}, \mathrm{CL}_{t}$, and $\mathrm{AR}_{t}$ \\
$\mathrm{E}$ & Generalized feedforward & Hyperbolic Tangent & 6-18-12-1 & $\mathrm{R}_{t}, \mathrm{RH}_{t}, \mathrm{WBT}_{t}, \mathrm{AP}_{t}, \mathrm{CL}_{t}$, and $\mathrm{AR}_{t}$ \\
$\mathrm{~F}$ & Generalized feedforward & Hyperbolic Tangent & 9-22-11-1 & $\mathrm{R}_{t}, \mathrm{RH}_{t}, \mathrm{WBT}_{t}, \mathrm{AP}_{t}, \mathrm{CL}_{t}, \mathrm{AR}_{t}, \mathrm{SR}_{t}, \mathrm{SR}_{t}, \mathrm{and} \mathrm{SR3}_{t}$ \\
$\mathrm{G}$ & Generalized feedforward & Hyperbolic Tangent & 9-22-11-1 & Same as Model F but only rainfall events \\
\hline
\end{tabular}

$\mathrm{R}_{t}=$ Rainfall intensity at $t, \mathrm{RH}_{t}=$ Relative humidity at $t, \mathrm{WBT}_{t}=$ wet bulb temperature at $t, \mathrm{AP}_{t}=$ Air pressure at $t, \mathrm{CL}_{t}=\mathrm{Cloudiness}$ at $t$, $\mathrm{AR}_{t}=$ Average hourly rainfall intensity of all rain gauges at $t$, and $\mathrm{SR} 1_{t}=$ Rainfall intensity at surrounding station 1 at $t$.

training set was used to calibrate the weights of the network; the cross validation set was used to monitor the progress of training process. The testing data set which used for the final evaluation of the model performance is the hourly rainfall records of the year 2003. Presented in this section are six distinctive alternative models, whose structure and result are considered as the most presentable for the preliminary test, details of these six ANN models are summarized in Table 1.

The first model (Model A) used the multilayer perceptron (MLP) network with a simple structure involving five nodes in the input layer, two hidden layers having 5 hidden nodes in each of the two layers, and one node in the output layer (5-5-5-1). Inputs to the model were hourly rainfall data (at time $t$ ) and past hourly rainfall with four hourly lag times i.e., from (t-4) to (t-1) at station E18, while the output is the rainfall intensity of the next hour $(t+1)$. The transfer function in nodes was the sigmoid function. For the second model (Model B), the network type, transfer function and input data were kept as same as Model A but the number of hidden nodes in both hidden layers was increased from 5 to 10 .

In the third model (Model C), the generalized feedforward network was employed, the input variables, transfer function and network architecture (5-10-10-1) were similar as Model B. The fourth model (Model D) also adopted the generalized feedforward network, with the sigmoid transfer function, but different input variables and model structure. Theoretically, the self-learning nature of ANN normally allows it to predict without extensive prior knowledge of all processes involved. However, a good understanding of the physical processes involved, and a hypothesis on how different processes (and their state variable) interact with each other would help in identifying parameters used in the input data. Thus, other meteorological parameters were added to the input data set and the past rainfall data was excluded as it brings more zero values to the training process (no rain periods). This resulted in six input variables for Model D, which included present time values of relative humidity, wet bulb temperature, air pressure, cloudiness, average hourly rainfall intensity of all the rain gauge stations, and present rainfall at the station E18. The model structure was modified to 6 input nodes, 16 and
12 hidden nodes in the first and second hidden layer, respectively, and 1 output node (6-16-12-1).

In the fifth model (Model E), the input data was remained as same as in Model D but the hyperbolic tangent function was used instead of the sigmoid function and the number of hidden node in the first layer was increased to 18 , which formed a new network architecture (6-18-12-1). In the sixth model (Model F), spatial data were also considered in the input variables for the ANN model, rainfall data of stations around E18 were included to the input that were used in Model D and E. A correlation analysis was carried out for 75 selected rain gauge stations to determine which stations are strongly related to the station E18. Results of the analysis revealed higher correlation of the stations E00, E19 and E26 with the station E18. Therefore, the present hourly rainfall data of these three stations were added to the input data set of Model E in the formulation of Model F. The change in the input variables resulted in an increase in the number of nodes in the input layer to 9 , increase in the number of nodes to 22 in the first hidden layer and 11 in the second hidden layer.

\section{Results and discussion}

\subsection{Comparison of models for one-hour ahead rainfall forecast at station E18}

The one-hour ahead forecast accuracy of the six ANN models in preliminary testing stage (model A to F) were evaluated using to Efficiency Index (EI), Root Mean Square Error (RMSE), and Correlation Coefficient $\left(R^{2}\right)$; the behavior of these parameters is presented in Table 2 . To investigate the models deeper in terms of quantitative forecast, all the patterns with no rain in observed data were excluded, and model performance indices based on the remaining data (rainy periods only) were re-evaluated and the results are shown in Table 3 .

It can be seen that there was consistency in the results obtained between the training and testing stages of these 

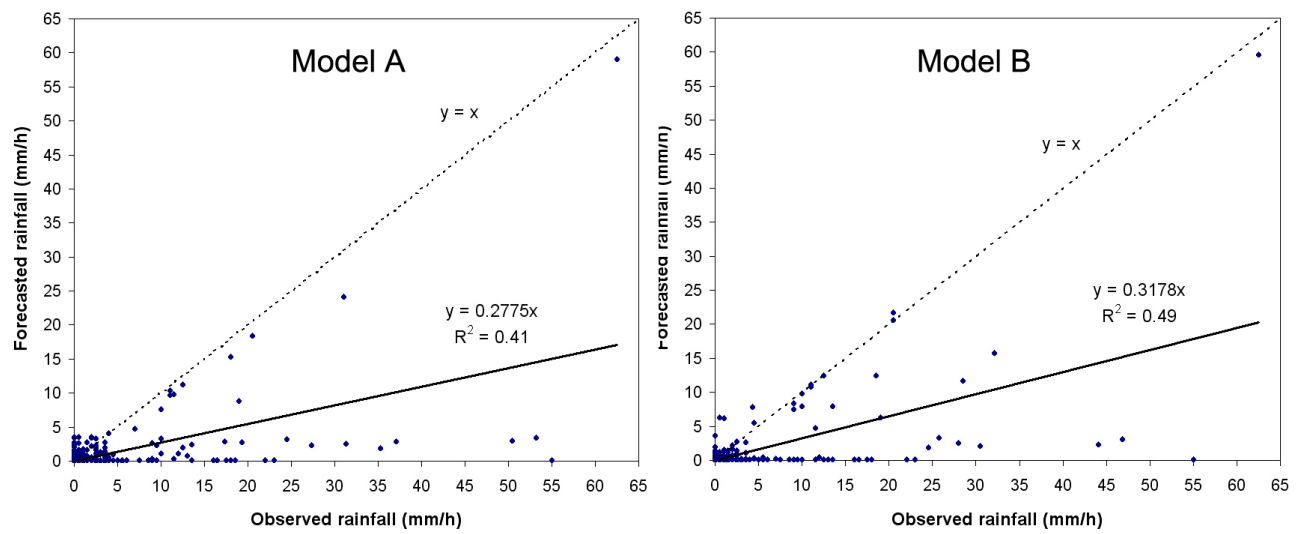

Fig. 4. Scatter plot of observed and forecasted rainfall for Model A and Model B (testing stage).
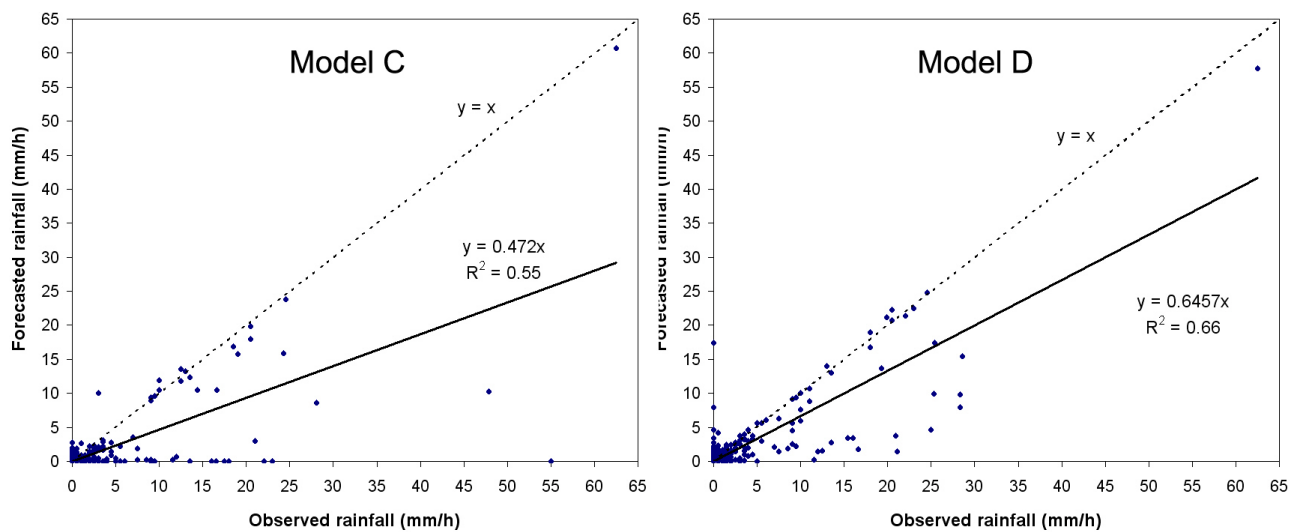

Fig. 5. Scatter plot of observed and forecasted rainfall for Model C and Model D (testing stage).

Table 2. Performance statistics of the six ANN models for $1 \mathrm{~h}$ rainfall forecasting at E18 station.

\begin{tabular}{lllllll}
\hline Model & A & B & C & D & E & F \\
\hline Index & \multicolumn{5}{c}{ Training } & $(1997,1998$ and 1999 data $)$ \\
EI $(\%)$ & 31.62 & 40.46 & 50.5 & 62.47 & 81.52 & 98.54 \\
RMSE $(\mathrm{mm} / \mathrm{h})$ & 2.15 & 1.96 & 1.75 & 1.51 & 0.98 & 0.53 \\
$R^{2}$ & 0.48 & 0.54 & 0.57 & 0.68 & 0.87 & 0.98 \\
EI $(\%)$ & 28.35 & 38.25 & 48.32 & 59.19 & 80.54 & 96.72 \\
RMSE $(\mathrm{mm} / \mathrm{h})$ & 2.1 & 1.98 & 1.86 & 1.56 & 1.05 & 0.71 \\
$R^{2}$ & 0.41 & 0.49 & 0.55 & 0.66 & 0.83 & 0.94 \\
\hline
\end{tabular}

models. This was attributed to the fact that the cross validation approach was helpful in detecting the best generalization point. The first three ANN models (A, B, C), which used only the characteristics of previous rainfall value as input variables, provided very low accuracy of forecast. The lowest score was obtained by Model A since the number of hidden nodes was insufficient to memorize and learn the pat- tern of training data. Although the number of hidden nodes was increased from 5 to 10 in each hidden layer in Model $\mathrm{B}$, improvement in the result was minor. Changing the network type in Model $\mathrm{C}$ enhanced the performance compared to Model A and B. This suggests that the generalized feedforward network performed better than the simple multilayer perceptron network in this study. Figures 4 and 5 depict the scatter plot of rainfall forecast against the observed record of these three models, showing that most low rainfalls are overestimated and high rainfalls are underestimated. These three models rendered several errors in forecasts; sometimes they provided rainfall forecasts when there was no rain. No rain in forecasted results versus no rain in the observed record was considered as a very high forecasting result; therefore, when the non-rainy period was excluded for quantitative investigation, the EI and coefficient of correlation were degraded as presented in Table 3. All these results of the first three models A, B and C (Tables 2 and 3) indicated that rainfallrainfall model structure is not working well in the approach of a continuous model.

Comparison of Model D with Model C, both of which used the same network type and transfer function but 
Table 3. Performance statistics of models for $1 \mathrm{~h}$ rainfall forecasting at E18 station (rainfall events only).

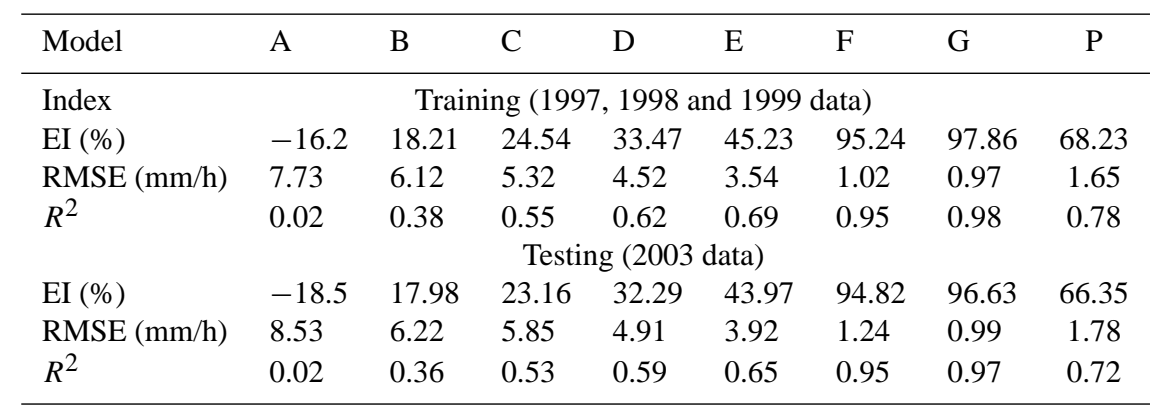
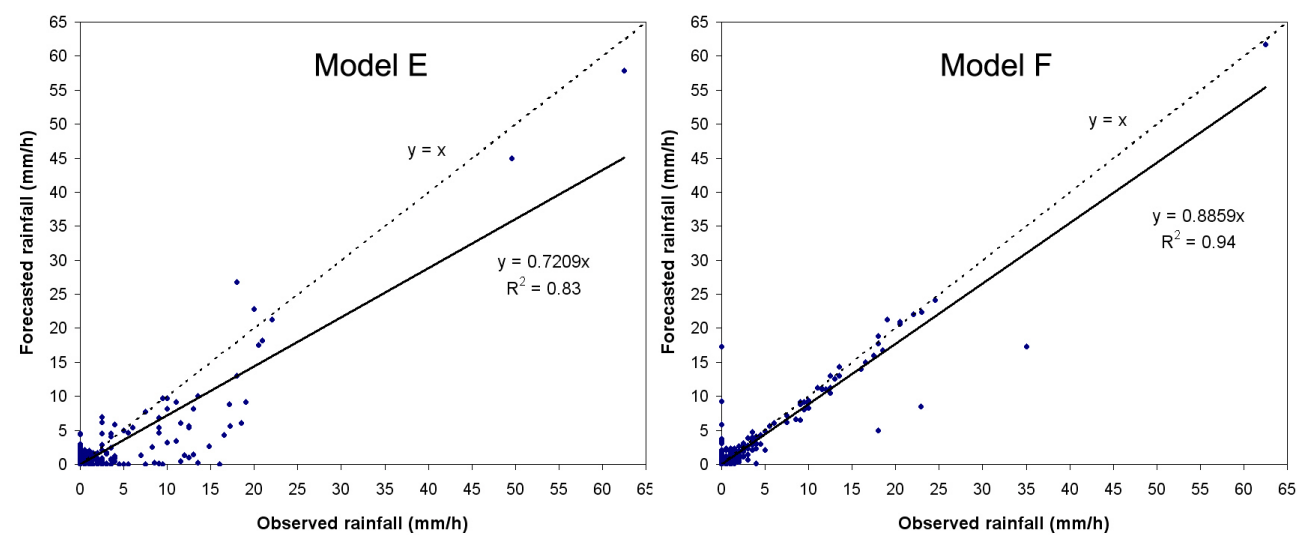

Fig. 6. Scatter plot of observed and forecasted rainfall for Model E and Model F (testing stage).

different input variables, brought a very interesting outcome. The combination of the meteorology information with rainfall series in the input data has generally improved model performance as the Model D yielded better results than Model $\mathrm{C}$ for both the training and testing phase. For model E, remarkable performance indicates that with hyperbolic tangent function, model $\mathrm{E}$ is capable of generalizing better results from the same set of input variables than model $\mathrm{D}$, which use the sigmoid function. Models $\mathrm{D}$ and $\mathrm{E}$ also show degraded performance indices, just as models A, B and C, when norain data was eliminated (Table 3). Figs. 5 and 6 reveal that both models $\mathrm{E}$ and $\mathrm{D}$ provided underestimated rainfall forecasts.

Model F, which involved the input data of rainfall at the station of forecast and the three surrounding stations, as well as other meteorological parameters (Table 1), produced the highest performance (Table 2). The scatter plot in Figure 6 confirms the match between forecasted and observed rainfall. Moreover, Model F also worked well when applied to each rainfall event (Table 3). The high accuracy result by Model $\mathrm{F}$ (Tables 2 and 3) again highlights the importance of using additional data to enable the ANN model to train and run in continuous mode.
In order to evaluate the superiority of the optimal model (Model F) over the conventional approach in the real time rainfall forecast, two other models were developed. The first model is Model G with the same setup as Model F, but using only rainfall events to feed in input as given in Table 1 . The second model is a persistence approach (Model P), or naïve prediction, widely used in forecasting theory. This predictive scheme, which simply substitutes the last measured values as the current forecast, represents a good bottom line benchmark.

The performance indices of Model G (Table 3) showed that in the first case, when applied with rainy data only, Model G works more or less comparably with Model F; similar results were obtained in the training stage and were slightly improved in the testing stage. However, in the second case, when Model $\mathrm{G}$ was applied in real time mode with continuous data of the year 2003, it was found that Model G produced a very large forecast error, as illustrated in the right panel of Fig. 7. Non-rainy periods included in the continuous data made this input set become unhomogeneous with the original training data (rainfall events only). Therefore, Model G, once trained with rainy data only, has no ability of generalization to unseen problems, and was unable to generate rainfall forecasts in continuous mode. 

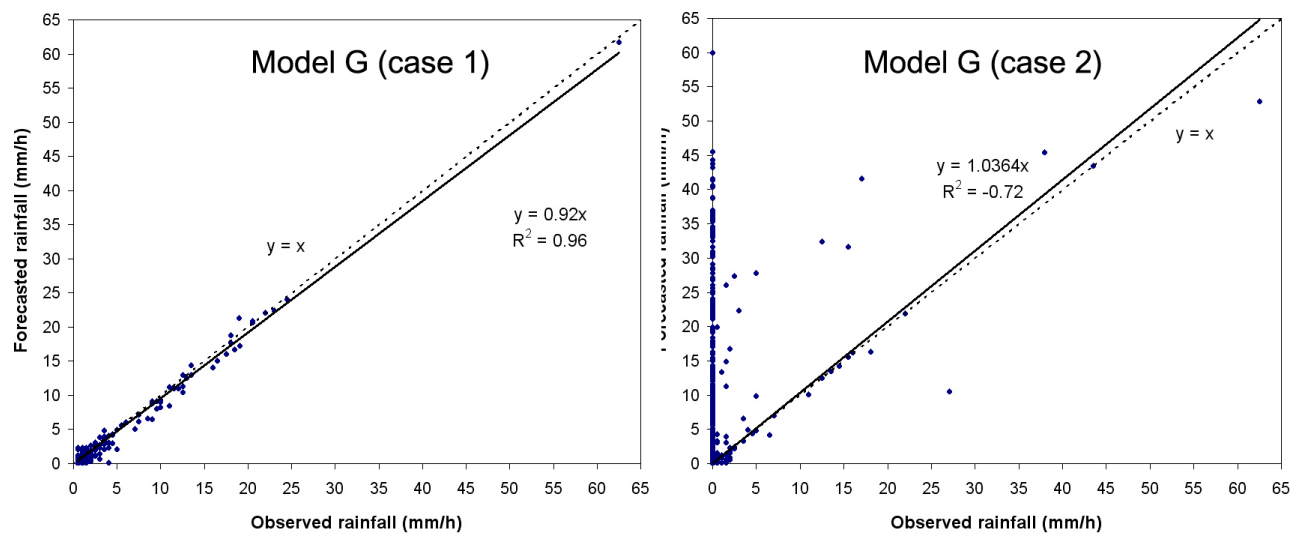

Fig. 7. Scatter plot of observed and forecasted rainfall for Model G (testing stage).

Table 4. Performance statistics for sensitivity analysis for $1 \mathrm{~h}$ rainfall forecasting at E18 station.

\begin{tabular}{|c|c|c|c|c|c|c|c|}
\hline Case & $\begin{array}{l}\text { Base case } \\
\text { (Model F) }\end{array}$ & $\begin{array}{l}\text { Without } \\
\text { relative humidity }\end{array}$ & $\begin{array}{l}\text { Without wet } \\
\text { bulb temperature }\end{array}$ & $\begin{array}{l}\text { Without } \\
\text { air pressure }\end{array}$ & $\begin{array}{l}\text { Without } \\
\text { cloudiness }\end{array}$ & $\begin{array}{l}\text { Without average rainfall } \\
\text { of all } 75 \text { stations }\end{array}$ & $\begin{array}{l}\text { Without rainfall at } \\
\text { surrounding } 3 \text { stations }\end{array}$ \\
\hline Index & \multicolumn{7}{|c|}{ Training (1997, 1998 and 1999 data) } \\
\hline EI $(\%)$ & 98.54 & 81.34 & 79.54 & 82.13 & 88.62 & 93.54 & 83.36 \\
\hline $\operatorname{RMSE}(\mathrm{mm} / \mathrm{h})$ & 0.53 & 0.94 & 1.12 & 0.92 & 0.82 & 0.68 & 0.91 \\
\hline$R^{2}$ & 0.98 & 0.85 & 0.82 & 0.87 & 0.92 & 0.96 & 0.89 \\
\hline \multicolumn{8}{|c|}{ Testing (2003 data) } \\
\hline $\mathrm{EI}(\%)$ & 96.72 & 80.32 & 76.96 & 81.54 & 85.41 & 92.15 & 82.51 \\
\hline $\operatorname{RMSE}(\mathrm{mm} / \mathrm{h})$ & 0.71 & 0.99 & 1.25 & 0.98 & 0.89 & 0.75 & 0.96 \\
\hline$R^{2}$ & 0.94 & 0.84 & 0.81 & 0.86 & 0.91 & 0.95 & 0.87 \\
\hline
\end{tabular}

Examination of Table 3 also reveals that the performance of the persistent model (Model P) gained the third place among eight presented models. However, since the persistent model considered the forecast equal to the last rainfall record, it always issued lag forecast and the performance indices of Model P were significantly less than that of Model $\mathrm{F}$ (Table 3). As a feature of a tropical climate, rainfall in Bangkok is rapid, with high fluctuation in both duration and intensity; therefore, the persistent approach is inadequate to provide applicable forecasts for real time purposes.

Comparison of Model $\mathrm{G}$ and persistent model with Model $\mathrm{F}$ confirmed that when applied for rainy data only, Model F had comparable performance with Model G and was much better than Model P. In the case of using continuous data, model F has outperformed both Model G and P.

\subsection{Sensitivity analysis}

While training a network, the effect that each of the network inputs has on the network output should be studied. This provides feedback as to which input parameters are the most significant. Based on this feedback, it may be decided to prune the input space by removing the insignificant parameters. This also reduces the size of the network, which in turn reduces the network complexity and the training time. The sensitivity analysis is carried out by removing each of the input parameters in turn from the input parameters used on Model $\mathrm{F}$ and then comparing the performance statistics, EI, RMSE and $R^{2}$. The greater the effect observed in the output, the greater is the sensitivity of that particular input parameter.

As mentioned earlier, the inputs into Model $\mathrm{F}$ included rainfall $(\mathrm{mm} / \mathrm{h})$ at the station $\mathrm{E} 18$, relative humidity $(\%)$, wet bulb temperature $\left({ }^{\circ} \mathrm{C}\right)$, air pressure $(\mathrm{HPa})$, total cloudiness, arithmetical average rainfall $(\mathrm{mm} / \mathrm{h})$ of all rain gauges, and rainfall $(\mathrm{mm} / \mathrm{h})$ from the three surrounding stations (strongly connected with station E18). The rainfall on the particular station was considered as the main parameter; hence this parameter was not included in the sensitivity analysis. The top three strongly connected stations (E00, E19 and E26) to E18 station were selected based on the correlation and were considered to have the same relative importance level as the correlation coefficients are $0.91,0.93$ and 0.87 , respectively. For this reason, in the sensitivity analysis, these three stations were excluded once, which formed an ANN structure (6-1812-1). For the remaining five cases, the same network architecture (8-22-11-1), using the hyperbolic tangent function and forecasting rainfall 1 hour ahead is used (Table 4).

As seen from Table 4, the ANN model without wet bulb temperature as input obtained the maximum EI reduction in the training stage. In the testing stage, the model without 


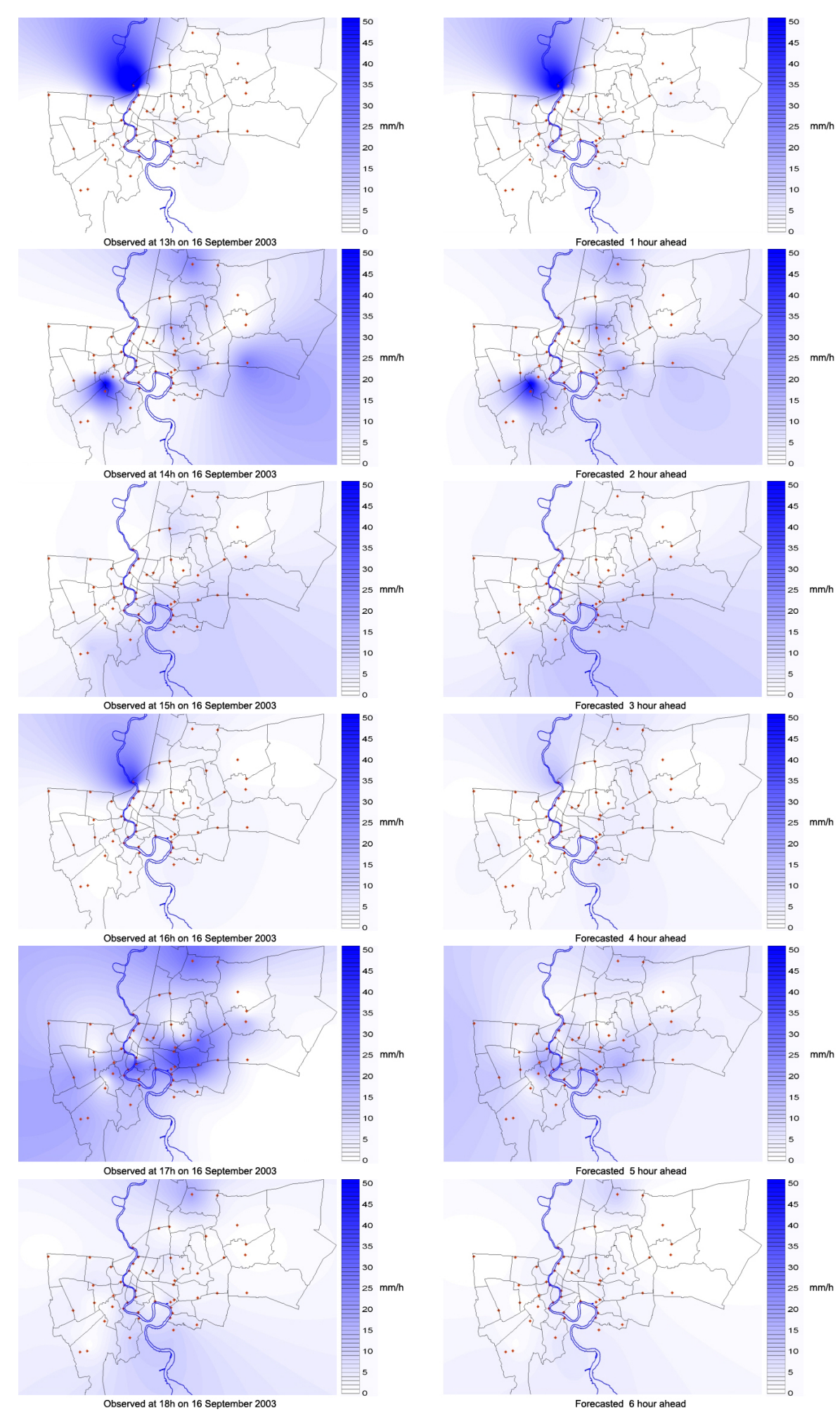

Fig. 8. Comparison of observed rainfall (left side maps) and predicted rainfall (right side maps) for 1 to $6 \mathrm{~h}$ ahead forecasting on 16 September 2003 (from 13:00 to 18:00). 
Table 5. Summary of ANN results for rainfall forecasting at 75 rainfall stations (Model F).

\begin{tabular}{llllllllll}
\hline Lead time & \multicolumn{3}{l}{ Efficiency index $(\%)$} & \multicolumn{3}{c}{ Correlation coefficient } & \multicolumn{3}{c}{ RMSE $(\mathrm{mm} / \mathrm{h})$} \\
\hline & Max & Min & Mean & Max & Min & Mean & Max & Min & Mean \\
1 h & 98 & 74 & 86 & 0.99 & 0.74 & 0.88 & 1.48 & 0.42 & 0.87 \\
2h & 87 & 63 & 69 & 0.92 & 0.63 & 0.77 & 2.16 & 0.73 & 1.36 \\
3h & 68 & 42 & 54 & 0.84 & 0.55 & 0.67 & 2.55 & 1.06 & 1.72 \\
4h & 62 & 35 & 45 & 0.78 & 0.48 & 0.64 & 2.82 & 1.11 & 1.85 \\
$5 \mathrm{~h}$ & 58 & 30 & 41 & 0.73 & 0.46 & 0.62 & 2.72 & 1.16 & 1.88 \\
$6 \mathrm{~h}$ & 48 & 29 & 36 & 0.71 & 0.36 & 0.60 & 2.75 & 1.24 & 1.93 \\
\hline
\end{tabular}

wet bulb temperature gave similar results: EI received the lowest value. This indicates that the most significant input is wet bulb temperature. Likewise, from overall performance of models in Table 4, the second most important parameter is the relative humidity, while other important parameters are the air pressure and the rainfall at the three surrounding stations. The cloudiness remained as the fifth most important parameter, with an EI decreasing to $88.62 \%$ for the model without this parameter. The ANN model without average rainfall of all stations gave EI of $93.54 \%$ in the training stage, nearest to the results of Model F, indicating that this input data contributed the least in improving the model results.

\subsection{Rainfall forecasting from 1 to $6 \mathrm{~h}$ for Bangkok area}

From the preliminary test, Model $\mathrm{F}$ was identified as the best model among six ANN testing models. The efficiency attained at $1 \mathrm{~h}$ ahead forecast is highly accurate; the scatter plot in Fig. 6 also shows that peaks forecast are matched with observed data. Therefore, Model F was selected to forecast rainfall at lead times of one to six hours at all 75 rain gauge stations in Bangkok. The same training approach and data selection of the preliminary test was implemented in the forecasting stage.

Table 5 presents the summarized ANN model results in terms of maximum, minimum, mean EI, $R^{2}$, and RMSE for rainfall forecasting from 1 to $6 \mathrm{~h}$ ahead at all 75 rain gauge stations. The models performed consistently well, providing stable and similar results for all stations. It is seen that the model performance decreases with the increasing lead time of the forecast. This is to be foreseen, as the ANN model uses a recursive method to forecast multi-step ahead. Thus the forecast errors are propagated and accumulated from step to step.

It can further be seen that the ANN models provide remarkably acceptable results of rainfall forecasts for 1 and $2 \mathrm{~h}$ ahead (Table 5). For $1 \mathrm{~h}$ lead forecasts at some stations the value of EI reached up to $98 \%$, while the lowest EI value of all stations was $74 \%$. Similarly, the maximum and minimum correlation coefficient values of 0.99 and 0.74 reflect highly satisfactory results. For $2 \mathrm{~h}$ ahead forecast, the results may also be considered quite satisfactory with maximum EI of $87 \%$, and minimum EI of $63 \%$; and maximum and minimum $R^{2}$ of 0.92 and 0.63 respectively. Forecasting results of $3 \mathrm{~h}$ ahead are less satisfactory, results for 4 to $6 \mathrm{~h}$ ahead may be considered to be poor with the mean EI varying between 45 and $36 \%$ and mean $R^{2}$ in the range from 0.78 to 0.71 .

Observed rainfall (left figures) and the predicted rainfall (right figures) for 1 to $6 \mathrm{~h}$ ahead forecasting on the rainfall event on 16 September 2003 are compared in Fig. 8. These maps are developed with the known geographical locations and the observed and forecasted rainfall at all 75 stations for clear visualization and comparison. The Kriging method was employed for interpolation of the scattered data over the study area.

As seen in Fig. 8, at 13:00 h on 16 September 2003, there was rainfall on few stations and the model could estimate $1 \mathrm{~h}$ ahead forecasts of rainfall quite accurately. Average measured rainfall is $0.78 \mathrm{~mm}$ and that of the forecast is $0.71 \mathrm{~mm}$, so the RMSE value of this forecast step is $0.65 \mathrm{~mm} / \mathrm{h}$. At 14:00 h, rain came from more stations in both the east and west side of Bangkok with an average observed amount of $2.5 \mathrm{~mm}$. The map based on the rainfall forecasted $2 \mathrm{~h}$ ahead corresponds reasonably well with the map developed based on the observed rainfall. In this step, the ANN model forecasted an average rainfall value of $1.95 \mathrm{~mm}$, and RMSE increased to $0.85 \mathrm{~mm} / \mathrm{h}$. Rainfall forecasts at $3 \mathrm{~h}$ ahead in this example was reasonably good as the shape of the rain map is matched between observed and forecasted. The RMSE value for this forecast step was $1.15 \mathrm{~mm} / \mathrm{h}$. Rainfall forecasts at $4 \mathrm{~h}$ ahead still formed a good rain map, except one station clearly provided a large underestimated rainfall forecast. The RMSE of this step was $1.42 \mathrm{~mm} / \mathrm{h}$. In both 5 and 6 hours forecasts, the ANN model gave erroneous forecast at several stations, and the RMSE value increased to $1.72 \mathrm{~mm} / \mathrm{h}$ and $1.83 \mathrm{~mm} / \mathrm{h}$ respectively. These illustrative results confirmed the findings presented in Table 5 that the rainfall forecast results for 1 to 3 hours ahead were satisfactory and for 4 to $6 \mathrm{~h}$ ahead were not acceptable and demand further improvements in techniques for longer lead time predictions. 


\section{Conclusions}

In this study, an Artificial Neural Network model was employed to forecast rainfall for Bangkok, Thailand, with lead times of 1 to $6 \mathrm{~h}$. Comparison of $1 \mathrm{~h}$ ahead rainfall forecast of the six models considered in the preliminary test showed that a combination of meteorological parameters such as relative humidity, air pressure, wet bulb temperature, and cloudiness, along with rainfall data at the forecasting station and other surrounding stations, as an input for the model could significantly improve the forecast accuracy and efficiency. Results of preliminary tests also concluded that the generalized feedforward network and hyperbolic tangent function performed well in this study. With the appropriate network architecture and especially with the use of auxiliary data, the ANN model was able to learn from continuous input data which contained both rain and dry periods, thus the model can be adopted to run for real time forecasting. The superiority in performance of the ANN model over that of the persistent model again confirmed that the real advantage of a continuous ANN model is that it can provide a satisfactory rainfall forecast at any moment.

It is important to determine the dominant model inputs, as this increases the generalization of the network for a given data. Furthermore, it can help reduce the size of the network and consequently reduce the training time. In this study, sensitivity analysis was used to rank the input parameters with respect to their importance in forecasting rainfall based on the model performance. Results of the sensitivity analysis indicated that the most important input parameter, besides rainfall itself, is the wet bulb temperature; further study over the entire rain gauge network could be carried out for more significant conclusions.

The ANN model was found to be efficient in fast computation and capable of handling the noisy and unstable data that are typical in the case of weather data. The predicted values of all 75 rain gauge stations matched well with the observed rainfall for forecasts with short lead times of 1 or 2 $\mathrm{h}$. Not only that, the rainfall forecasting for $3 \mathrm{~h}$ ahead using the ANN model also provided reasonably acceptable results. The efficiency indices were gradually reduced as the forecast lead time increased from 4 to $6 \mathrm{~h}$. Although the model performance of 6 hour forecasting was low and the forecasting was not as accurate as expected, the developed model can still be used for practical applications such as rainfall forecasting and flood management for the urban areas.

Acknowledgements. This article is a part of doctoral research conducted by the first author at Water Engineering and Management, Asian Institute of Technology, Bangkok, Thailand. The financial support provided by the DANIDA for pursuing the study is gratefully acknowledged. The author would like to express sincere gratitude to the staff of the Thai Meteorological Department and the Bangkok Metropolitan Administration for providing, sourcing and facilitating access to and usage of invaluable data and information used in this study. Thanks are also extended to the anonymous reviewer and the editor for their constructive contributions to the manuscript.

Edited by: E. Toth

\section{References}

Abrahart, R. J. and See, L.: Comparing neural network and autoregressive moving average techniques for the provision of continuous river flow forecast in two contrasting catchments, Hydrol. Proc., 14, 2157-2172, 2000.

Ahmad, S. and Simonovic, S. P.: An artificial neural network model for generating hydrograph from hydro-meteorological parameters, J. Hydrol., 315(1-4), 236-251, 2005.

ASCE: Task Committee on Application of Artificial Neural Networks in Hydrology. I: Preliminary Concepts, J. Hydrol. Eng., 5(2), 115-123, 2000.

ASCE: Task Committee on Application of Artificial Neural Networks in Hydrology. II: Hydrologic Applications, J. Hydrol. Eng., 5(2), 124-137, 2000

Campolo, M. and Soldati, A.: Forecasting river flow rate during low-flow periods using neural networks, Water Resour. Res., 35 (11), 3547-3552, 1999.

Coulibaly, P., Anctil, F., and Bobee, B.: Daily reservoir inflow forecasting using artificial neural networks with stopped training approach, J. Hydrol., 230, 244-257, 2000.

Fletcher, D. S. and Goss, E.: Forecasting with neural network: An application using bankruptcy data, Inf. Manage., 24, 159-167, 1993.

French, M. N., Krajewski, W. F., and Cuykendall, R. R.: Rainfall forecasting in space and time using neural network, J. Hydrol., 137, 1-31, 1992.

Gwangseob, K. and Ana, P. B.: Quantitative flood forecasting using multisensor data and neural networks, Journal of Hydrology, 246, 45-62, 2001.

Hsu, K., Gupta, H. V., and Sorooshian, S.: Artificial neural network modeling of the rainfall-runoff process, Water Resour. Res., 31(10), 2517-2530, 1995.

Koizumi, K.: An objective method to modify numerical model forecasts with newly given weather data using an artificial neural network, Weather Forecast., 14, 109-118, 1999.

Lapedes, A. S. and Farber, R. M.: Nonlinear signal processing using neural networks: Prediction and system modeling, Los Alamos Report LA-UR 87-2662, 1987.

Lippmann, R. P.: An introduction to computing with neural nets, IEEE ASSP Magazine, 4, 4-22, 1987.

Luk, K. C., Ball, J. E., and Sharma, A.: A study of optimal model lag and spatial inputs to artificial neural network for rainfall forecasting, J. Hydrol., 227, 56-65, 2000.

Maier, R. H. and Dandy, G. C.: The use of artificial neural network for the prediction of water quality parameters, Water Resour. Res., 32(4), 1013-1022, 1996.

Maier, R. H. and Dandy, G. C.: Comparison of various methods for training feed-forward neural network for salinity forecasting, Water Resour. Res., 35(8), 2591-2596, 1999.

Rogers, L. L. and Dowla, F. U.: Optimization of groundwater remediation using artificial neural networks with parallel solute transport modeling, Water Resour. Res., 30(2), 457-481, 1994. 
Rosenblatt, F.: The Perceptron: A Probabilistic Model for Information Storage and Organization in the Brain, Cornell Aeronautical Laboratory, Psychological Review, 65(6), 386-408, 1958.

Rumelhart, D. E. and McClelland, J. L.: Parallel Distributed Processing: Explorations in the Microstructure of Cognition, London, UK, The MIT Press, 1986.

Shamseldin, A. Y.: Application of a neural network technique to rainfall-runoff modeling, J. Hydrol., 199, 272-294, 1997.

Toth, E., Montanari, A., and Brath, A.: Comparison of short-term rainfall prediction model for real-time flood forecasting, J. Hydrol., 239, 132-147, 2000.
Zealand, C. M., Burn, D. H., and Simonovic, S. P.: Short term streamflow forecasting using artificial neural networks, J. Hydrol., 214, 32-48, 1999.

Werbos, P. J.: Beyond Regression: New Tools for Prediction and Analysis in Behavioral Sciences, Ph.D. dissertation, Appl. Math., Harvard University, Cambridge, MA, USA, 1974. 